$\xi=0$

\title{
Quasi-linear correlation between high-frequency and static die-lectric constants in II-VI and III-V semiconductors
}

\author{
Salah Daoud ${ }^{1 *}$, Abdelhalim Bencheikh ${ }^{2}$, Laarbi Belagraa ${ }^{1,3}$ \\ ${ }^{1}$ Laboratory of Materials and Electronic Systems (LMSE), Faculty of Sciences and Technology, Mohamed Elbachir El Ibrahimi, \\ Bordj Bou Arreridj University, Bordj Bou Arreridj (34000), Algeria \\ ${ }^{2}$ Département des Sciences et techniques, Université Mohamed Elbachir El Ibrahimi de Bordj Bou Arreridj, \\ Bordj Bou Arreridj (34000), Algérie \\ ${ }^{3}$ Civil Engineering Department, Faculty of Technology, Mohamed Boudiaf University, M'sila (28000), Algeria \\ *Corresponding author E-mail: salah_daoud07@yahoo.fr
}

\begin{abstract}
A quantitative form of the linear correlation between the high-frequency and static dielectric constants in $A^{\mathrm{N}} \mathrm{B}^{8-\mathrm{N}}(\mathrm{N}=2,3)$ tetrahedrally coordinated semiconductor materials, and also in I-VII group alkali halides was studied. So, a quasi-linear relationship was found between the high-frequency and the static dielectric constants for some selected II-VI (ZnS, ZnSe, ZnTe and CdTe) and III-V (AlP, AlAs, $\mathrm{AlSb}, \ldots .$. etc) cubic zincblende type materials, in the other side a weak uphill linear relationship has been found in the case of I-VII (LiF, $\mathrm{NaF}, \mathrm{LiCl}, \ldots$...tc) group alkali halides compounds. In the case of II-VI and III-V cubic zincblende semiconductors, the linear regression is established with a correlation coefficient $(\rho)$ of about 0.98 . The significance of the linear regression is given as the probability $P$ $<0.0001$ of the null hypothesis.
\end{abstract}

Keywords: Frequency and the Static Dielectric Constants; II-VI and III-V Semiconductors; Alkali Halides Compounds.

\section{Introduction}

$\mathrm{A}^{\mathrm{N}} \mathrm{B}^{8-\mathrm{N}}$ binary semiconductor compounds have been extensively studied in recent years, because of their technological importance and their crystallographic structure [1]. The optical properties of a semiconductor are one of the key parameters for materials used in solar cell and optoelectronic devices manufacturing (laser diodes, detectors, etc...) [1]. Furthermore, the dielectric constant of these materials are of fundamental importance for the behavior of charge carriers, dopants, point defects (vacancies), linear defects (dislocations) and impurities in both semiconductors and insulators [1]. Knowledge of the high-frequency dielectric constant and other optical properties of semiconducting materials help in the improvement of the manufacturing of the solar cells and other optoelectronic devices by the good choice of the appropriate materials to the appropriate applications.

Due to technological importance of I-VII alkali halides solids, IIVI and III-V semiconductor compounds, several works on their electronic, optical, mechanical and thermal properties were recently published in the literature [2-10].

In the object to predict the Debye temperature $\theta_{\mathrm{D}}$ and melting point $\mathrm{T}_{\mathrm{m}}$ of cubic II-VI and III-V semiconductors, Kumar et al. [2] have given six simple relations between these two parameters and some other physical quantities. And in the object to compare their work to the others presented in the literature, they found lower percentage deviations, which show a significant improvement over other empirical relations proposed by earlier workers. In the other hand the Optoelectronic properties of zincblende and wurtzite structured binary solids have been studied by Yadav et al. [3].

Verma et al. [4] used a new approach utilizing the concept of ionic charge theory to explain the inherent properties such as lattice thermal conductivity and bulk modulus of III-V and II-VI semiconductors. They found that the lattice thermal conductivity of these semiconductors exhibit a linear relationship when plotted on a log-log scale again the nearest-neighbor distance.

In our recent work [8], we report first principles calculations of the near-neighbor distance and the average energy gap using the pseudopotential plane wave method, in the framework of the density functional theory (DFT), then the optoelectronic properties such as homopolar and heteropolar energies, dielectric constant and optical susceptibility and some other thermal properties of boron-bismuth (BBi) compound in its zincblende phase have been determined by using several formulas of the literature.

In our very recent work [12], we have improved an expression related the optical refractive index and optical electronegativity difference of $\mathrm{A}^{\mathrm{N}} \mathrm{B}^{8-\mathrm{N}}(\mathrm{N}=1,2,3)$ tetrahedrally coordinated semiconductor materials.

To study the electronic properties of boron based pnictides compounds, Bouhafs et al. [13] have also performed an ab initio calculation, and they found that the electronic properties of these materials shows a particular features which differ from those of the other usual III-V semiconductors. Varshney et al. [14] have investigated the pressure effect on the elastic properties, and sound velocity of boron based pnictides in zincblende and $\mathrm{NaCl}$ phases. Due to a very importance of the optical and electronic properties in optoelectronic devices manufacturing, more analyses and discussion of these quantities becomes necessary.

In the present paper, we attempt to discuss the linear correlation between the high-frequency $\varepsilon_{\infty}$ and static $\varepsilon_{0}$ dielectric constants in $\mathrm{A}^{\mathrm{N}} \mathrm{B}^{8-\mathrm{N}}(\mathrm{N}=2,3)$ tetrahedrally coordinated semiconductor materials, and also in I-VII group alkali halides compounds. 


\section{Theory, results and discussion}

\subsection{Cubic zincblende crystal structure}

Most used materials in electronics and optoelectronics fields have an underlying face-centered cubic (FCC) lattice. However, in zincblende (prototype: cubic of zinc and sulfur atoms) crystal structure, the materials (example: GaAs, AlAs, CdS, CdTe, etc.,) have two different atoms per basis; the coordinates of the two basis atoms are $(0,0,0)$ and $(1 / 4,1 / 4,1 / 4)$ respectively [15]. In cubic zincblende crystal structure, the different atoms (zinc and sulfur) atoms are tetrahedrally coordinated. Figure 1 shows the different positions occupied by each atom in cubic zincblende crystal structure. If the two atoms of the basis constitute the solid are identical (example: $\mathrm{Si}, \mathrm{Ge}, \mathrm{C}$, etc...,), the crystal structure is called cubic diamond [15].

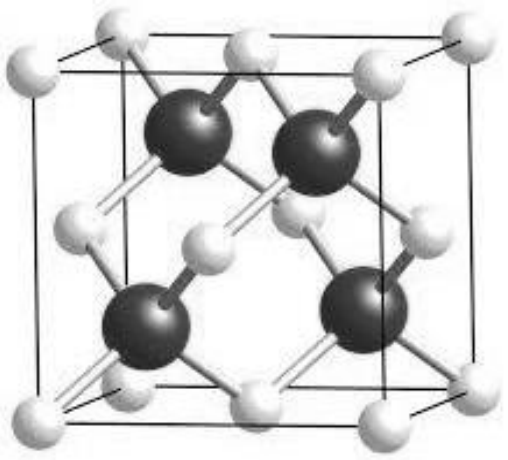

Fig. 1: Position Occupied by Each Atom in Cubic Zincblende Structure. The Structure Consists of the Interpenetrating FCC Lattices, One Displaced from the other by A Distance $(1 / 4,1 / 4,1 / 4)$ Along the Body Diagonal [15].

\subsection{Lydanne - sachs -teller relation}

Lydanne - Sachs -Teller relation [16] is a formula which relies the high-frequency and static dielectric constants of crystals, it was firstly used in studying some cubic crystals with only one IRactive mode. So it has played an important role in the investigations of long wavelength infrared active optical phonons [17]. The dielectric function can be expressed as function of the frequency $\omega$ as follows [18].

$$
\varepsilon(\omega)=\varepsilon_{\infty}\left(1+\left(\mathrm{S} /\left(\omega_{\mathrm{TO}}^{2}-\omega^{2}\right)\right)\right)
$$

Where: $\mathrm{S}=\omega_{\mathrm{LO}}^{2}-\omega_{\mathrm{TO}}^{2}$ is the polarization constant. This splitting of the optical modes is converted to a difference in low- and highfrequency constants.

Where: $\omega_{\mathrm{LO}}$ and $\omega_{\mathrm{TO}}$ are the frequencies of the longitudinal and transverse optical modes respectively.

By substituting $\mathrm{S}=\omega_{\mathrm{LO}}^{2}-\omega_{\mathrm{TO}}^{2}$ in Eq. (01), the latter can be written as follows [18].

$$
\varepsilon(\omega)=\varepsilon_{\infty}\left(1+\left(\left(\omega_{\mathrm{LO}}^{2}-\omega_{\mathrm{TO}}^{2}\right) /\left(\omega_{\mathrm{TO}}^{2}-\omega^{2}\right)\right)\right)
$$

To obtain a relation between the high-frequency dielectric constants $\varepsilon_{\infty}$ and the static one $\varepsilon_{0}$, we substitute $\omega=0$ in Eq. (02), the latter constants become related by the following expression [18, 19].

$\left(\varepsilon_{0} / \varepsilon_{\infty}\right)=\left(\omega_{\mathrm{LO}}^{2} / \omega_{\mathrm{TO}}^{2}\right) \Rightarrow \varepsilon_{\infty}=\varepsilon_{0}\left(\omega_{\mathrm{TO}}^{2} / \omega_{\mathrm{LO}}^{2}\right)$
This latter expression describes in simplified form the relation between the high-frequency $\varepsilon_{\infty}$ and static $\varepsilon_{0}$ dielectric constants in crystals.

\section{Results and discussion}

The aim of this work is to study the linear correlation between the high-frequency and static dielectric constants for some selected IIVI and III-V cubic zincblende semiconductors and I-VII alkali halides. The term "correlation" refers to a process for establishing whether or not relationships existing between two variables. The correlation coefficient $\rho$ measures the strength and direction of a linear relationship between two variables on a Scatterplot. The value of $\rho$ is always between +1 and -1 (For more detail on The correlation coefficient, see for example: Elementary Statistics for Geographers, Third Edition, by, James E. Burt, Gerald M. Barber, David L. Rigby, Guilford Publications (2009)). The experimental values of the high-frequency $\varepsilon_{\infty}$ and static $\varepsilon_{0}$ dielectric constants of zincblende structured solids reported in table 1 are taken from the Ref [1], and of Alkali halides reported also in table 1 are taken from the Ref [19] and references cited therein.

Table 1: High-Frequency $\varepsilon_{\infty}$ and Static $\varepsilon_{0}$ Dielectric Constants of ZincBlende Structured Solids [1], and Alkali Halides Solids [19]

\begin{tabular}{llllll}
\hline $\begin{array}{l}\text { Comp } \\
\text { ounds }\end{array}$ & $\varepsilon_{\infty}[1]$ & $\varepsilon_{0}[1]$ & $\begin{array}{l}\text { Comp- } \\
\text { ounds }\end{array}$ & $\varepsilon_{\infty}[19]$ & $\varepsilon_{0}[19]$ \\
\hline $\mathrm{ZnS}$ & 5.13 & 8.32 & $\mathrm{LiF}$ & 1.9 & 8.9 \\
$\mathrm{ZnSe}$ & 6.2 & 9.2 & $\mathrm{LiCl}$ & 2.7 & 12 \\
$\mathrm{ZnTe}$ & 7.28 & 9.67 & $\mathrm{LiBr}$ & 3.2 & 13.2 \\
$\mathrm{CdTe}$ & 7.21 & 10.76 & $\mathrm{NaF}$ & 1.7 & 5.1 \\
$\mathrm{AlP}$ & 7.54 & 9.8 & $\mathrm{NaCl}$ & 2.25 & 5.9 \\
$\mathrm{AlAs}$ & 8.16 & 10.06 & $\mathrm{NaBr}$ & 2.6 & 6.4 \\
$\mathrm{AlSb}$ & 10.24 & 12.04 & $\mathrm{NaI}$ & 3 & 7.3 \\
$\mathrm{GaP}$ & 9.11 & 11.11 & $\mathrm{KF}$ & 1.5 & 5.5 \\
$\mathrm{GaAs}$ & 10.88 & 12.85 & $\mathrm{KCl}$ & 2.1 & 4.85 \\
$\mathrm{GaSb}$ & 14.44 & 15.69 & $\mathrm{KBr}$ & 2.3 & 4.9 \\
$\mathrm{InP}$ & 9.61 & 12.655 & $\mathrm{KI}$ & 2.7 & 5.1 \\
$\mathrm{InAs}$ & 12.25 & 15.16 & $\mathrm{RbF}$ & 1.9 & 6.5 \\
$\mathrm{InSb}$ & 15.68 & 17.88 & $\mathrm{RbCl}$ & 2.2 & 4.9 \\
& & & $\mathrm{RbBr}$ & 2.3 & 4.9 \\
& & & $\mathrm{RbI}$ & 2.6 & 5 \\
& & & $\mathrm{CsCl}$ & 2.6 & 7.2 \\
& & & $\mathrm{CsBr}$ & 2.8 & 6.5 \\
& & & $\mathrm{CsI}$ & 3 & 5.65 \\
\hline
\end{tabular}

Starting by the scatter-plot of the high-frequency $\varepsilon_{\infty}$ and static $\varepsilon_{0}$ dielectric constants for some selected II-VI and III-V cubic zincblende materials; the results are presented in figure 2 . From the latter, it can be seen clearly the quasi-linear relationship (a strong uphill linear relationship) between the high-frequency $\varepsilon_{\infty}$ and static $\varepsilon_{0}$ dielectric constants. The linear fit of this later can be written as follow.

$\varepsilon_{\infty}=\mathrm{a}+\mathrm{b} \varepsilon_{0}$

Where $\mathrm{a}$ and $\mathrm{b}$ are constants.

The numerical values of the constants $a$ and $b$ for selected II-VI and III-V cubic zincblende materials are: 3.5 and 0.87 respectively. The linear regression is established with a correlation coefficient $\rho=0.98$. The significance of the linear regression is given as the probability $\mathrm{P}<0.0001$ of the null hypothesis (probability that there is no correlation). 


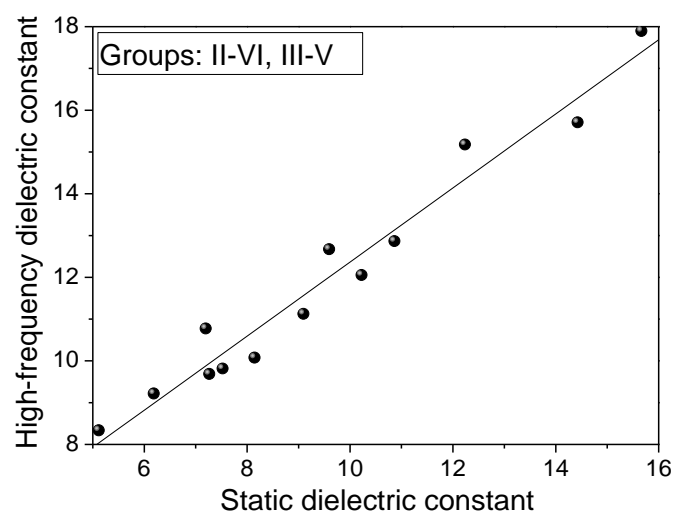

Fig. 2: Scatterplot Of $\varepsilon_{\infty}$ and $\varepsilon_{0}$ Data, plus Fitted Straight Line in II-VI and III-V Zincblende Compounds.

The high-frequency $\varepsilon_{\infty}$ and static $\varepsilon_{0}$ dielectric constants for alkali halides solids are plotted in Figure 3, where it is observed a weak uphill linear relationship between the high-frequency $\varepsilon_{\infty}$ and static $\varepsilon_{0}$ dielectric constants of these materials. From the data of the Table 1 and the Figure 3, we observe clearly that the lithium compounds $\mathrm{LiX}(\mathrm{X}=\mathrm{F}, \mathrm{Cl}, \mathrm{Br})$ posses the higher $\varepsilon_{\infty}$ compared to other I-VII alkali halides. So it is possible to improve the linear correlation in I-VII alkali halides materials by separating the $\mathrm{LiX}(\mathrm{X}=\mathrm{F}$, $\mathrm{Cl}, \mathrm{Br}$ ) compounds and other materials.

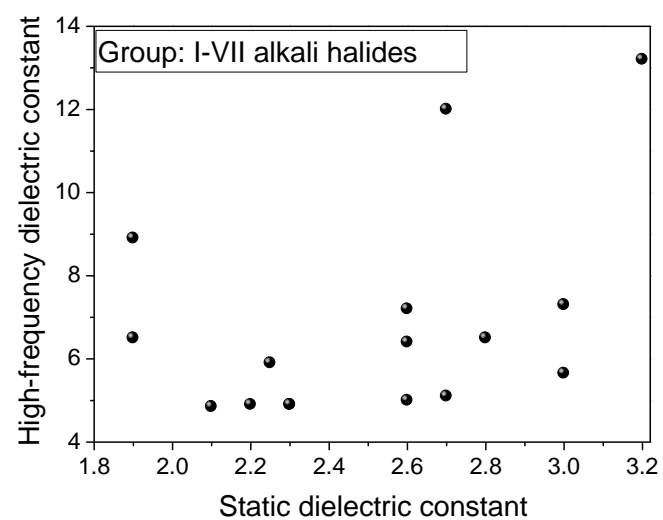

Fig. 3: Scatterplot Of $\varepsilon_{\infty}$ and $\varepsilon_{0}$ Data in I-VII Alkali Halides Compounds.

\section{Conclusion}

In the present paper it is shown that a quasi-linear relation is found between the high-frequency and static dielectric constants in $\mathrm{A}^{\mathrm{N}} \mathrm{B}^{8-\mathrm{N}}(\mathrm{N}=2,3)$ tetrahedrally coordinated semiconductor materials. The linear regression is established with a correlation coefficient $(\rho)$ of about 0.98 . The significance of the linear regression is given as the probability $\mathrm{P}<0.0001$ of the null hypothesis. Whereas a weak uphill linear relationship has been found in the case of I-VII group alkali halides compounds. Perhaps the principal cause of these different behaviors is due to the nature of bonds (degree of covalency and ionicity of ions formed the compounds) and the crystallographic structures (zincblende, rock-salt and $\mathrm{CsCl}$ ) which are different in these two types of materials.

\section{References}

[1] A. S. Verma, Naresh Pal, B K Sarkar, R Bhandari, V. K. Jindal, "Dielectric constants of zincblende semiconductors", Physica Scripta, Vol.85, No.1, (2012) pp. 015705 (4pp). and references cited therein. http://dx. doi:10.1088/0031-8949/85/01/015705.

[2] V. Kumar, V. Jha, A. K. Shrivastava, "Debye temperature and melting point of II-VI and III-V semiconductors", Crystal Research and
Technology, Vol.45, No.9, (2010), pp. 920-924. http://dx.doi.org/ 10.1002/crat.201000268.

[3] D. S. Yadav, C. kumar, J. Sigh, Parashuram, G. Kumar, "Optoelectronic properties of zinc blende and wurtzite structured binary solids", Journal of Engineering and Computer Innovations, Vol.3, No.2, (2012), pp. 26-35. http://www.academicjournals.org/journal/ JECI/article-abstract/DC4DD528645 https://doi.org/10.5897/JECI12.005.

[4] A. S. Verma, B. K. Sarkar, V. K. Jindal, "Inherent properties of binary tetrahedral semiconductors", Physica B, Vol.45, (2010) pp. 1737-1739. http://dx.doi.org/10.1016/j.physb.2010.01.029.

[5] S. Adachi, Properties of Group-IV, III-V and II-VI Semiconductors, John Wiley \& Sons, Chichester, (2005).

[6] S. Daoud, "Sound velocities and thermal properties of BX (X=As, $\mathrm{Sb}$ ) compounds", International journal of scientific world, Vol.3, No.1, (2015), pp. 43-48. http://dx.doi.org/10.14419/ijsw.v3i1.4039.

[7] N. Paliwal, V. Srivastava, A. K. Srivastava, "Electronic band structure and heat capacity calculation of some TIX $(\mathrm{X}=\mathrm{Sb}, \mathrm{Bi}) \mathrm{com}-$ pounds", Advances in materials physics and chemistry, Vol.6, (2016), pp. 47-53. http://dx.doi.org/10.4236/ampc.2016.63005.

[8] S. Daoud, N. Bioud, N. Lebgaa, R. Mezouar, "Optoelectronic and thermal properties of boron- bismuth compound", International Journal of Physical Research, Vol.2, No.2, (2014), pp. 27-31. http://dx.doi.org/10.14419/ijpr.v2i2.2760.

[9] S. Daoud, N. Bioud, "Structural properties of (B3) TIP under pressure", International Journal of Physical Research, Vol.2, No.2, (2014), pp. 50-55. http://dx.doi.org/10.14419/ijpr.v2i2.3100.

[10] S. Daoud, "Comment on" Structural phase transition, electronic and elastic properties in $\operatorname{TlX}(\mathrm{X}=\mathrm{N}, \mathrm{P}$, as $)$ compounds: Pressureinduced effects" [Comput. Mater. Sci. 50 (2010) 203-210]", Computational Materials Science : Vol.111, (2016), pp. 532 - 533 http://dx.doi.org/10.1016/j.commatsci.2015.09.022.

[11] M. Ustundag, M. Aslan, Battal G. Yalcin, "The first-principles study on physical properties and phase stability of Boron-V (BN, $\mathrm{BP}, \mathrm{BAs}, \mathrm{BSb}$ and $\mathrm{BBi}$ ) compounds", Computational Materials Science, Vol.81, (2014), pp. 471-477.

[12] A. Latreche, S. Daoud, "Modified expression for calculating refractive index of $\mathrm{A}^{\mathrm{N}} \mathrm{B}^{8-\mathrm{N}}$ type binary semiconductors ", International Journal of Physical Research, Vol.4, No.2, (2016), pp. 48-51.

[13] B. Bouhafs, H. Aourag, M. Cartier, "Trends in band-gap pressure coefficients in boron compounds BP, BAs, and BSb", Journal of Physics: Condensed Matter, Vol.12, No.26, (2000), pp. 5655-5668 http://iopscience.iop.org/0953-8984/12/26/312. https://doi.org/10.1088/0953-8984/12/26/312.

[14] D. Varshney, G. Joshi, M. Varshney, S. Shriya, "Pressure induced mechanical properties of boron based pnictides", Solid State Sciences, Vol.12, No.5, (2010), pp. 864-872.

[15] Jasprit Singh, Electronic and Optoelectronic Properties of Semiconductor Structures, University of Michigan, Ann Arbor, Cambridge University Press, (2003).

[16] R.M. Lyddane, R.G. Sachs, E.Teller, "On the polar vibrations of alkali halides", Physical Review, Vol.59, No.8, (1941), pp. 673676. http://journals.aps.org/pr/abstract/10.1103/PhysRev.59.673. https://doi.org/10.1103/PhysRev.59.673.

[17] A.S. Chaves, S.P.S. Porto, "Generalized Lyddane-Sachs-Teller relation", Solid State Communications, Vol.13, No.7, (1973), pp. 865868. http://www.sciencedirect.com/science/article/pii/00381098739 03864.

[18] D. K. Kerry, Semiconductors, New York: Macmillan Publishing Company, USA, (1991). Print ISBN: 0-02-946519-2.

[19] E. Burstein, S. Perkowitz, M. Brodsky, "The dielectric properties of the cubic IV-VI compound semiconductors", Journal de Physique Colloques, (1968), 29(C4), pp.C4-78-C4-83. And references cited therein. https://hal.archives-ouvertes.fr/jpa-00213615. 